\title{
TRATAMIENTO ENDODÓNTICO A UNA PIEZA CON ALTERACIÓN MORFOLÓGICA DENS IN DENTE REPORTE DE CASO CLÍNICO
}

\section{DENS IN DENT ENDODONTIC TREATMENT CLINICAL CASE REPORT}

\author{
Villavicencio-Caparó Emilio ${ }^{1}$ \\ ${ }^{1}$ Especialidad de Cariología y Endodoncia. Universidad Católica de Santa María Arequipa-Perú. \\ *emiliovc82@hotmail.com
}

\begin{abstract}
Resumen
Introducción: La alteración de la forma de los dientes es una complicación anatómica en cuanto estas piezas requieran tratamiento de conducto radicular, ya que los exámenes convencionales no aportan mucho para el diagnóstico y la planificación, es necesario utilizar la tecnología para poder resolver dichos casos. Reporte de Caso: Se presenta el caso de un paciente de sexo masculino, con patología de necrosis pulpar y absceso apical crónico de la pieza 2.1, la cual al diagnóstico clínico se pudo determinar que tenía alteración de la forma (dens in dente) y al estudio radiográfico se obtiene información insuficiente del grado de invaginación en la raíz, lo cual dificulta la planificación del tratamiento endodóntico, así mismo el paciente estaba en tratamiento de ortodoncia por lo cual requería rehabilitar de forma permanente la pieza dental. En este caso el uso de la tomografía axial computarizada fue de mucha utilidad para el diagnóstico y la planificación del tratamiento radicular, la cual permitió tener información real de la longitud del diente, la presencia de dos conductos separados a nivel medio y apical de la raíz, pudiendo tener un protocolo de desinfección y obturación previsibles para el éxito del tratamiento. Conclusión : La implementación de nuevos métodos de diagnóstico utilizados para tratamientos dentales como la Tomografía Axial Computarizada (TAC), ayuda a poder resolver casos cada vez más complejos con una alta tasa de éxito a diferencia de los métodos tradicionales que por sus limitaciones dan información insuficiente para este tipo de tratamientos.
\end{abstract}

Palabras clave: Dens in dente, TAC.

\begin{abstract}
Introduction: The alteration of the shape of the teeth is an anatomical complication when these pieces require root canal treatment, since conventional examinations do not contribute much for diagnosis and planning, it is necessary to use the technology to solve such cases. Case Report: The case of a male patient with pulpal necrosis and chronic apical abscess of the item 2.1 is presented, which at clinical diagnosis could be determined to have altered form (dens in tooth) and to Radiographic study is obtained insufficient information of the degree of invagination in the root, which makes difficult the planning of the endodontic treatment, also the patient was in orthodontic treatment for which it required to permanently rehabilitate the dental piece. In this case the use of computed axial tomography was very useful for diagnosis and planning of root treatment, which allowed to have real information on tooth length, the presence of two separate ducts at the middle and apical root, Being able to have a protocol of disinfection and obturation predictable for the success of the treatment. Conclusion: The implementation of new diagnostic methods used for dental treatments such as Computerized Tomography $(C T)$, helps to solve increasingly complex cases with a high success rate unlike traditional methods that because of their limitations give insufficient information For this type of treatments.
\end{abstract}

Key words: Dens in dente, TAC.

\section{INTRODUCCIÓN}

La presencia de anomalías de la formación de los dientes es un factor que complica el tratamiento endodóntico de una pieza dental, ${ }^{1}$ la variación del número de conductos o la presencia de canales accesorios que no pueden ser determinados solo con la radiografía convencional puede provocar errores de la planificación del procedimiento y llevar al fracaso del tratamiento por dejar espacios radiculares sin tratar, por lo que la utilización de mejores exámenes auxiliares como la Tomografía Axial Computarizada TAC 
pueden ayudar a desarrollar un protocolo previsible y al éxito del tratamiento en favor de los pacientes.

El Dens in dente es una anomalía de forma que tiene una prevalencia de $19.5 \%$ en la población, ${ }^{2}$ es producida en los primeros momentos de formación del germen dentario, dando como resultado una invaginación del diente en el proceso de calcificación. ${ }^{3}$ El uso de la TAC ayuda al estudio y planificación del procedimiento, porque permite conocer con detalle la forma del diente a tratar, pudiendo tener una estrategia más clara para poder abordar el caso de una mejor forma.

\section{REPORTE DE CASO}

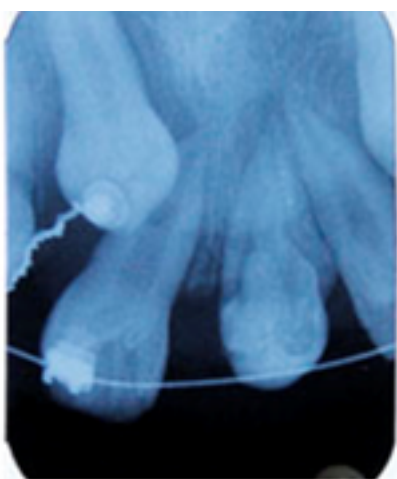

a.

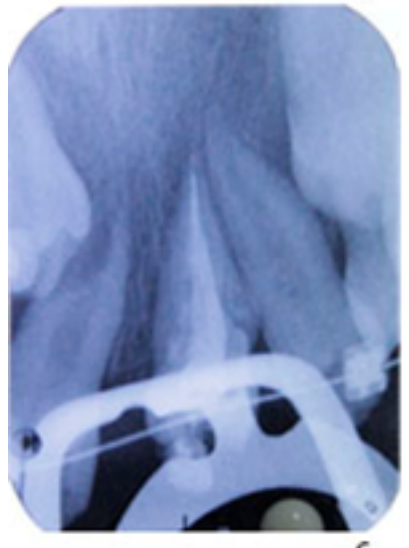

c.

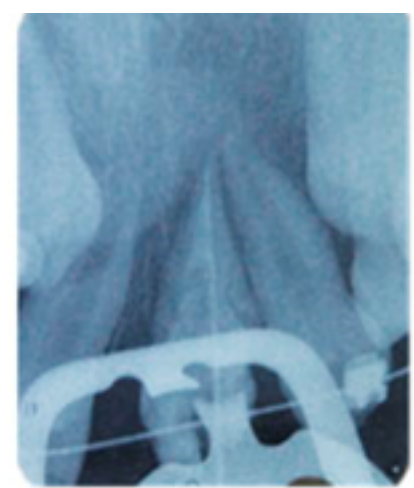

b.

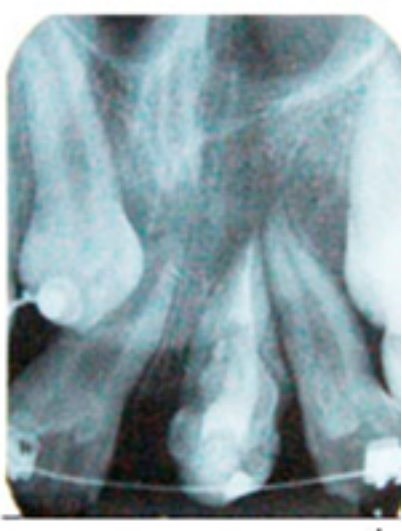

d.
Fig. 1. a: radiografía de diagnóstico, b: radiografía de conductometria, c: radiografía de obturación, d: radiografía de control 3 meses.

Paciente de sexo masculino de 9 años de edad es referido al área de segunda especialidad de endodoncia de la Facultad de Odontología de la Universidad Católica de Santa María de Arequipa, por presentar un absceso a nivel de la pieza 2.1; a la observación clínica, el paciente presentaba un diente ya tratado con una restauración de resina compuesta, una fístula a nivel del ápice del diente compatible con un absceso apical crónico y tratamiento ortodóntico en proceso.

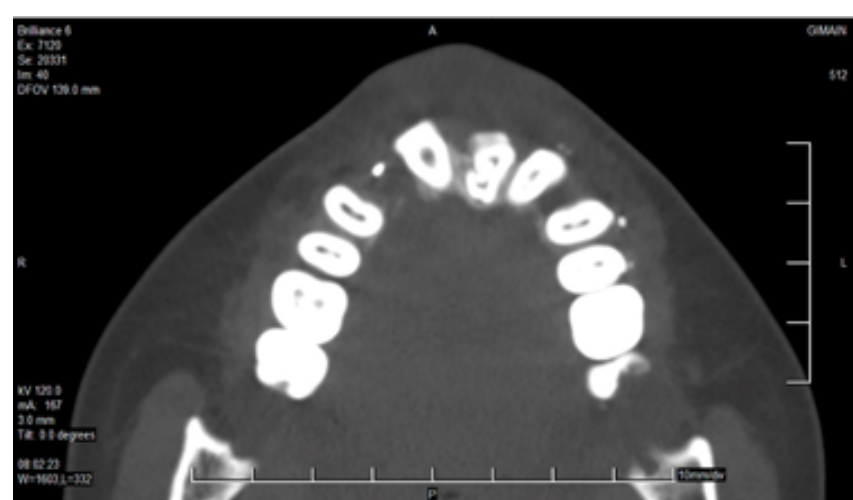

Fig. 2. TAC PRIMER CORTE

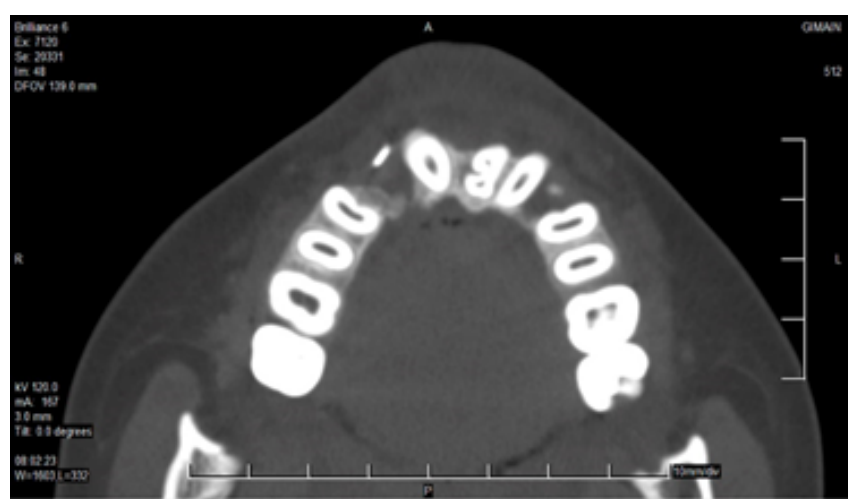

Fig. 3. TAC SEGUNDO CORTE

En la evaluación clínica la pieza presentaba dolor leve a la percusión vertical y respuesta negativa a los test de vitalidad, la forma de la corona clínica tenía alteración. Se tomó una radiografía periapical de diagnóstico (Figura 1 a) en la cual se podía observar a nivel del ápice una imagen radiolúcida, en la raíz en el sistema de conductos no se podía determinar la cantidad de canales radiculares pudiendo observarse 2 o 3 en la misma raíz, y presentando una cámara pulpar amplia. El diagnóstico de la pieza fue registrado como necrosis pulpar, con absceso dento alveolar crónico. ${ }^{4}$ Para lograr un plan de tratamiento más certero se pidió al paciente una TAC en la cual pudimos observar que la invaginación del diente iniciaba en el tercio cervical de la pieza dental (Figura 2), se extendía por el tercio medio de la raíz donde también se aprecia el inicio de la destrucción ósea provocada por el proceso infeccioso (Figura 3) y a nivel apical se observa los dos conductos con forámenes independientes y el final de la invaginación (figura 4). Tomando todos estos datos se pudo determinar fehacientemente que la anomalía de forma del diente corresponde a un dens in dente tipo III-a, según clasificación de Oehlers. ${ }^{5}$

La tomografía también en un corte sagital del diente nos 


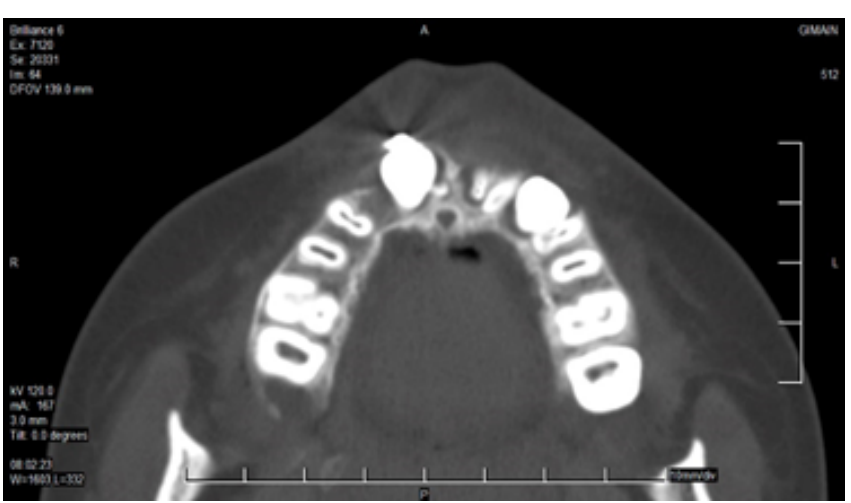

Fig. 4. TAC TERCER CORTE

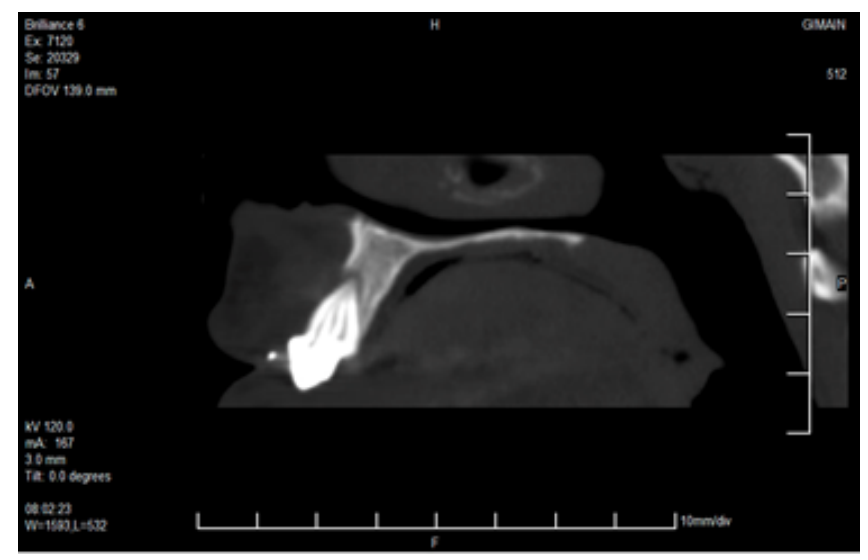

Fig. 5. TAC PRIMER CORTE SAGITAL

muestra que la invaginación simula un conducto más (Figura 5) lo que se podía observar en la radiografía de diagnóstico, pero con el corte transversal esto queda desvirtuado ya que se complementa la información determinando la presencia de dos conductos independientes y la invaginación a nivel central de la pieza, dicha anomalía pudo dar la oportunidad de cometer el error de buscar un tercer conducto. Otro dato importante recopilado de la tomografía fue la longitud real del diente, para utilizarla en referencia a la longitud real de trabajo, la cual se realizó digitalmente en el software de visualización de TAC determinando el eje principal del diente obteniendo una medida de $21.1 \mathrm{~mm}$ (Figura 6).

El tratamiento se inició colocando anestesia infiltrativa en la línea cero anatómica a nivel del diente a tratar, se procedió a realizar el aislamiento absoluto y luego la apertura cameral por la superficie incisal del diente para tener acceso recto al sistema de conductos, ya que el acceso habitual es modificado por la anomalía anatómica, se removió la restauración en camino al techo de la cámara pulpar, al ingresar al sistema de conductos ya teniendo la planificación para proceder a la instrumentación de la pieza.

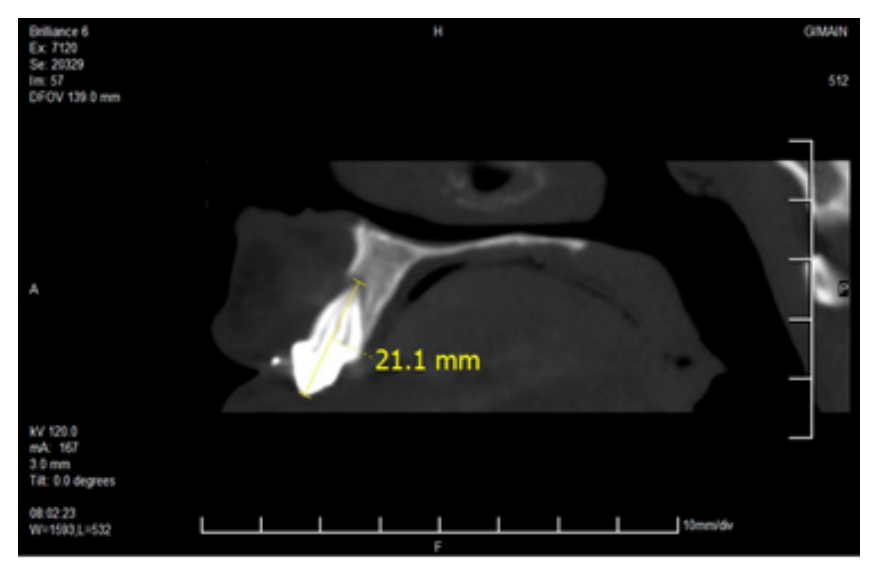

Fig. 6. TAC SEGUNDO CORTE SAGITAL

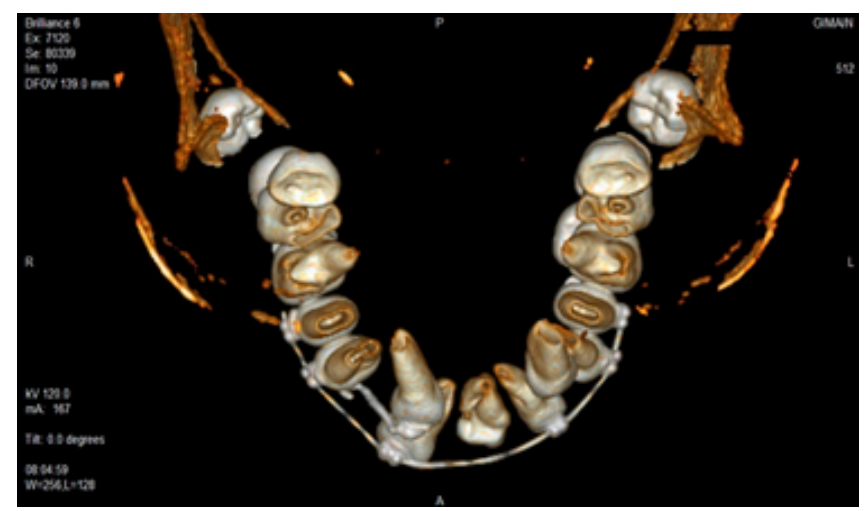

Fig. 7. RECONSTRUCCIÓN 3D CON TAC VISTA APICAL

Se procedió a realizar la permeabilización de los conductos utilizando una lima 10 e irrigación constante de hipoclorito de sodio al $5.25 \%$; para lograr la conductometria utilizamos un sistema de localizador electrónico de ápice Mini Apex Locator de la marca Sybro Endo (Figura 1 b), obteniendo la medida real de trabajo de $20.5 \mathrm{~mm}$ para ambos conductos y determinando como instrumento memoria la Lima 35, la técnica de instrumentación utilizada fue "Crown Down", se trabajó los conductos y colocamos medicación de hidróxido de calcio para obturar en una segunda cita.

Para la segunda cita luego de 7 días de iniciar la endodoncia, la fístula que presentaba la pieza a nivel del ápice ya estaba en proceso de recuperación y el paciente refirió que la molestias desaparecieron. Se procedió a realizar el retiro de la medicación y realizar la obturación con un cemento a base de hidróxido de calcio empleando la técnica Hibrida de Tagger ${ }^{6}$ (Figura 1 c). Se hizo el control radiográfico final que fue realizado a los 3 meses donde se puede observar que la lesión ósea está remitiendo y el pronóstico para el diente en cuanto a ser rehabilitado es 


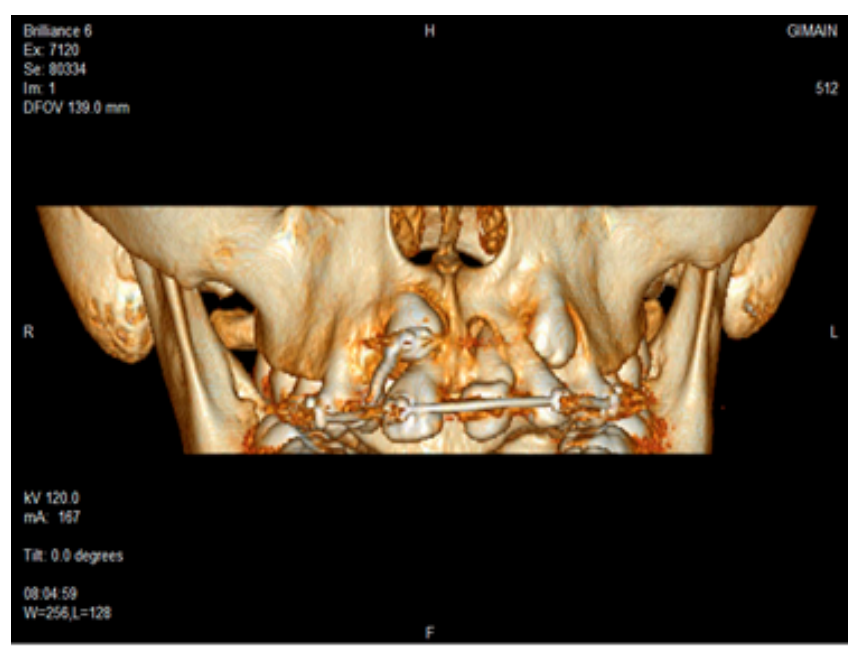

Fig. 8. RECONSTRUCCIÓN 3D CON TAC VISTA FRONTAL

favorable (Figura $1 \mathrm{~d}$ ).

\section{DISCUSIÓN}

En el presente caso clínico se utilizó la radiografía como método de apoyo al diagnóstico, ${ }^{7}$ al realizar la conductometría y la conometría, se presentó un reto muy importante, dado que la forma y longitud del conducto, se presentó con características inusuales. ${ }^{8-10}$ La tomografía fue de utilidad para planificar y ejecutar la conformación del conducto radicular, en cuanto a la obturación del conducto se realizó con los materiales de uso habitual en la práctica de la especialidad, ${ }^{11}$ en la Universidad Católica de Santa María de Arequipa y dentro de su programa de segunda especialidad. Se realizó seguimiento al caso a la semana, dos semanas, al mes y a los 3 meses de acuerdo a las sugerencias de la evidencia disponible. ${ }^{5,12}$

\section{CONCLUSIÓN}

El tratamiento de dientes permanentes con anomalías de forma, presentan una mayor dificultad al diagnóstico y realización de los procedimientos, en cuanto podamos utilizar la tecnología y saber interpretarla para realizar una planificación del caso, podemos tener resultados favorables y predecibles que favorecen a los pacientes en la permanencia de piezas naturales por un largo periodo de tiempo.

Conflicto de intereses y financiamiento El autor declara no tener conflicto de intereses, haber cumplido con los requisitos de autoría y haber autofinanciado este artículo

\section{Referencias}

1 Bhuvaneswari BB, Shivakumar B. A Variant Form of Dens Invaginatus in Permanent Maxillary Canine. International
Journal of Dental Sciences and Research. 2014; 2(4B): p. 1-3.

2 Casimiro-Iriarte S, Caballero-García S, Geller-Palti D. Asociación del Dens in Dente con el Diente en Pala y Diente Cónico en Piezas Anteriores. Odontología Pediátrica. 2016 Julio - Diciembre; 14(2): p. 16 - 25.

3 Khan S KSBVBRLK. Dens Invaginatus: Review, relevance, and report of 3 cases. J Dent Child. 2012; 79(3): p. 143 - 53.

4 American Association of Endodontists (AAE). AAE Consensus Conference Recommended Diagnostic. Journal of Endodontic. 2009 Diciembre; 35(12): p. 1634.

5 Barzuna M. Dens in dente: Anomalía dental difícil de tratar. Revista Científica Odontológica. 2013 Diciembre; 9(2): p. 35-38.

6 De Lima M. Endodoncia de la Biología a la Técnica Sao Paulo: AMOLCA; 2009.

7 Durack C, Patel S. The use of cone beam computed tomography in the management of dens invaginatus affecting a strategic tooth in a patient affected by hypodontia: a case report. International Endodontic Journal. 2011 mayo; 44(5): p. 474-83.

8 Aguiar CM, Ferreira JP, Câmara AC\&dFJA. Type 2 dens invaginatus in a maxillary lateral incisor: a case report of a conventional endodontic treatment. Journal of clinical pediatric dentistry. 2008; 33(2): p. 103-6.

9 Pritesh Kisanlal A, Jyoti W, Manjusha W. A Rare Case of Type III Dens Invaginatus in a Mandibular Second Premolar and Its Nonsurgical Endodontic Management by Using Cone-beam Computed Tomography: A Case Repor. Journal of Endodontics. 2016 Abril; 42(4): p. 669-672.

10 Bansal M, Singh NN, Singh AP. A rare presentation of dens in dente in the mandibular third molar with extra oral sinus. Journal of Oral \& Maxillofacial Pathology. 2010; 14(2): p. 80-82.

11 Calderón Gutiérrez AK, Villarreal Escamilla LA, Juárez García NR, Flores Treviño JJ, López Martínez F. Tratamiento endodóntico de un dens in dente. Revista Mexicana de Estomatología. 2015 Diciembre; 2(3): p. 24-25.

12 Heydari A, Rahmani M. Treatment of Dens Invagination in a Maxillary Lateral Incisor. Iranian Endodontic Journal. 2015; 10(3): p. 211-213.

Recibido: 10 de Octubre de 2016

Aceptado: 11 de Marzo de 2017 